\title{
Awareness and perception of dentistry students studying in Cyprus regarding COVID-19: A cross-sectional study
}

\author{
Cenk Serhan Özverela,b, Sevcan Kurtulmus-Yilmazc
}

\author{
${ }^{a}$ Department of Basic Medical Sciences, Faculty of Dentistry, Near East University, Lefkosa, Cyprus, Mersin10, Turkey \\ ${ }^{b}$ Near East University, DESAM Institute, Lefkosa, Cyprus, Mersin10, Turkey \\ cDepartment of Prosthodontics, Faculty of Dentistry, Near East University, Lefkosa, CyprusMersin10, Turkey \\ Corresponding Author: Cenk Serhan Özverel (E-mail: cenkozverel@gmail.com ) \\ (Submitted: 05 August 2020 - Revised version received: 11 August 2020 - Accepted: 27 August 2020 - Published online: 30 October 2020)
}

\begin{abstract}
:
Objectives: The present study aimed to evaluate the awareness and assessing the knowledge of dental students and dentists on COVID-19. Methods: The study population consisted of undergraduate students who are currently studying dentistry in Cyprus. Students were sent an online survey and data were collected on 16 April. Survey comprised of questions about the demography, education level of the participants, their infection control knowledge, and conditions that require treatment during pandemic.

Results: A total of 594 students with equal gender distribution joined the survey. The age group of the participants were mostly between 18 and 25 age (94\%) with 63\% pre-clinical and 37\% clinical students. The majority of participants were aware that dentists were at highrisk group in terms of COVID-19 (98.5\%). When clinical questions were asked, data indicated strong relationship between participants' responses with their level of study. Upon asking COVID-19-specific questions, participants that have taken microbiology course were reported to have higher correct answer rate.

Conclusions: Dental students studying in Cyprus demonstrated high awareness of COVID-19 and the higher risks of COVID-19 exposure that dental professionals face. Study demonstrated the importance of microbiology courses, as it significantly affected the answers to the questions on diagnosis methods and immune response to COVID-19.

Keywords: dentistry students, survey, dental education, coronavirus, COVID-19, SARS-CoV-2
\end{abstract}

\section{Introduction:}

A novel type of coronavirus has been recognized at the beginning of December 2019. ${ }^{1}$ The virus was initially named as 2019-CoV which was later called severe acute respiratory syndrome coronavirus 2 (SARS-CoV-2). ${ }^{2}$ The epidemic was first started in Wuhan, China in December 2019. COVID-19 was found to be a zoonotic pathogen according to a recent research with pangolins and Chinese horseshoe bats, similar to the severe acute respiratory syndrome (SARS) coronavirus and Middle East respiratory syndrome (MERS) coronavirus. ${ }^{3-5}$ As of 15 June, COVID-19 has been recognized in more than 215 countries, with a total of over 24 million laboratory-confirmed cases and more than 441,000 deaths. ${ }^{6}$ This novel viral infection spreads faster and has higher contagious rate than SARS and MERS. ${ }^{5}$ According to studies, the incubation period of COVID-19 was estimated between 1 and 14 days. ${ }^{1,7}$ The transmission route of COVID-19 to human was reported to be via air-borne droplets, blood-borne droplets, saliva, body secretions, touching, hand-shaking, and close contact with infected person as well as virus contaminated surfaces. In addition to these transmission routes, fecal-oral route could carry a risk as coronavirus was detected in the stool of patients. ${ }^{8,9}$

The majority of patients with COVID-19 represent mild clinical manifestations with fever, shortness of breath, fatigue, muscle pain, headache, diarrhea, vomiting, and dry cough. ${ }^{9-11}$ In some severe conditions, development of pneumonia, acute respiratory distress syndrome, shock, and arrhythmia were investigated. Patients with severe conditions were kept under consent of intensive-care units..$^{9-13}$

The most susceptible individuals were found to be immune-compromised individuals with older age and people with existing clinical conditions such as diabetes, hypertension, and cardiovascular diseases. These susceptible individuals were associated with severe clinical manifestations and poor prognosis. ${ }^{13-15}$

The travel history of patients 14 days prior to symptom onset was regarded as an important parameter in terms of diagnosis of COVID-19. Apart from the travel history, various laboratory tests [e.g., reverse transcriptase polymerase chain reaction (RT-PCR), chest computed tomography (CT), serological tests] were applied to patients with clinical symptoms in order to diagnose the disease. Until now, there is no specific anti-nCoV treatment, so majority of the treatment methods applied were supportive treatments. ${ }^{16}$ All these findings suggested the importance of early diagnosis and self-isolation in terms of COVID-19.

COVID-19 was found to be very contagious to some of the professions, especially the health-care workers. Huge numbers of health-care workers were reported to have acquired COVID-19 while working with COVID-19 infected individuals. ${ }^{17}$ These findings indicate that dental practitioners were also under high risk of getting COVID-19 due to their working environments (close contact with patients, usage of high speed rotary instruments, expose to body fluids such as saliva and blood). ${ }^{5}$ Although COVID-19 patients were not allowed to have dental treatments, some asymptomatic cases, or individuals with post infection would carry a huge risk in terms of these dental practitioners. ${ }^{3,18}$ These unavoidable contacts with patients during this pandemic period made dentists to have an extreme awareness and integrity to deal with disease and manage the control of its spread. 
The presence of infection prevention guidelines by country's ministry of health provide minimum requirements that are needed to be applied by health-care workers. Following these precautions precisely would help patients' protection and also the dental professionals. This study aimed to evaluate the awareness and assessing the knowledge of dental students and dentists on COVID-19.

\section{Materials and Methods:}

\section{Sample}

A total of 1370 undergraduate students who are currently studying dentistry in Cyprus were invited to take part in an online questionnaire. Invitation was posted on student's class representatives and students' social media groups. The period of the survey was April 15-16, 2020.

\section{Materials}

Questions were developed following the current guidelines ${ }^{16}$ and literature ${ }^{19}$ regarding COVID-19. An online Google Forms was used to construct a questionnaire comprising the following areas: (1) Demographic questions (gender, age, ethnicity), (2) questions related to their education year (pre-clinical or clinical classes, whether they have taken microbiology courses), (3) general questions to determine their COVID-19 knowledge, (4) questions related to diagnosis of COVID-19, (5) questions to measure their infection control knowledge, and (6) questions related to COVID-19 and dental applications. 'No opinion' option has also been added to some of the suitable questions asked during the survey.

All participants were informed about the questionnaire beforehand. Participation of respondents was voluntary and anonymous. Ethical approval was obtained from the Institutional Ethics Committee of Near East University (YDU/2020/79-1105).

\section{Data Analysis}

Data were collected in an Excel file and statistical analysis was done on SPSS 16.0 (IBM Corp, New York, USA). Chi-square and Spearman correlation tests were performed to assess the relation of the dentistry students' responses with respect to gender and to their clinical experience (according to their education level) and whether they have taken microbiology courses.

\section{Results:}

\section{Demography and Education}

This study covers a total of 594 dentistry students (291 females and 303 males), with a responsive rate of $49.5 \%$ of total invited students. The age of participants were ranged from 18 to $40+$, with most common group belonging to $18-25$ ages $(94.5 \%, 561)$. The vast majority of survey participants are people who come from international countries such as; Turkey $(69 \%, 410)$, Iran $(5 \%, 30)$, Jordan $(3 \%, 18)$, Iraq $(4 \%, 24)$, Syria $(4 \%, 24)$, United Arab Emirates $(2 \%, 12)$, Lebanon $(2 \%, 12)$, and Germany, France, Canada, Nigeria, Sudan with $<1 \%$. International students were also asked whether they returned to their home countries after the start of COVID-19 and 47\% (280) of the participants reported that they have returned to their countries. The majority of students (40\%) who turned back their countries indicated that they wanted to be with their families during the pandemic (Fig. 1). The participants of survey were also asked which classes they went to during the academic year 2019-2020, so that the differences between pre-clinical and clinical classes were aimed to take into account. According to the data, 375 (63\%) participants were in pre-clinical classes (1st, 2nd and 3rd years) and 219 (37\%) participants were clinical classes (4th and 5th years). Participants' microbiology knowledge was also assessed during the survey and 64\% (343) of the participants reported that they had microbiology classes during their dentistry education.

\section{Awareness of Participants}

The vast majority of respondents said they were first aware of COVID-19 pandemic through TV news $(43 \%, 261)$ and social media $(51 \%, 301)$. Only small population of participants was first aware of COVID-19 pandemic through newspapers (2\%, 12 ) and university announcements $(4 \%, 24)$. Participants were asked about the sources of information they are following to be aware of COVID-19 status. The main platform participants used was social media $(35 \%, 208)$, TV News $(26 \%, 154)$, the ministry of health website $(21 \%, 125)$, and to a lesser extent, research journals related to COVID-19 $(12 \%, 71)$ were reported. Participants' awareness were also investigated by asking current worldwide status (April 15) of COVID-19 pandemic. Almost all of the participants knew the country where pandemic originated $(99.5 \%, 591)$. The majority defined the country with the highest number of death rate as USA $(70 \%, 416)$ and the country with the highest recovery rate as China $(53 \%, 315)$.

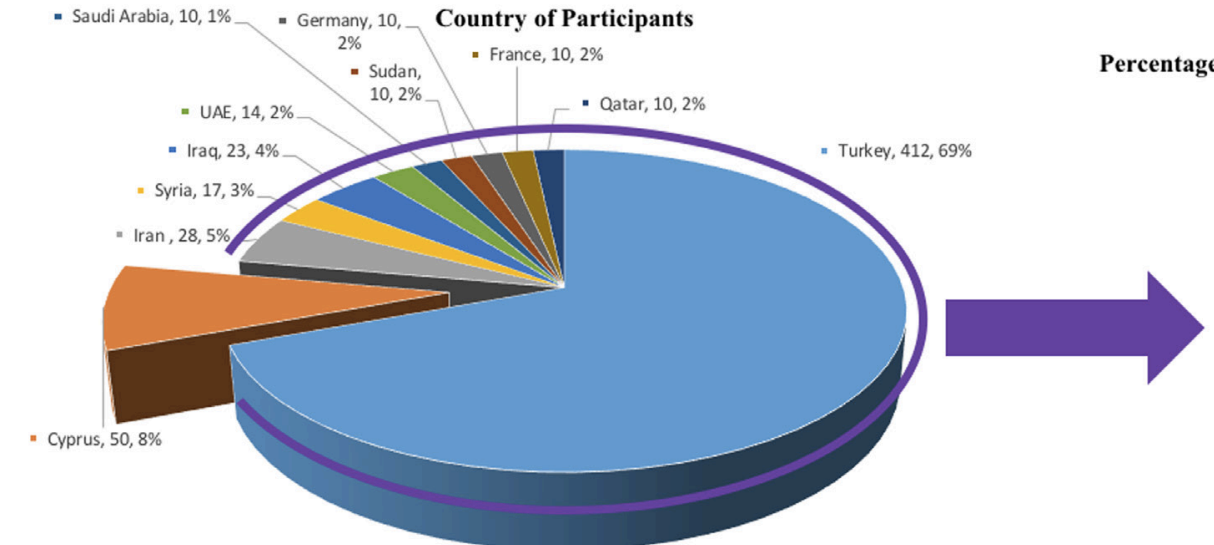

ercentage of foreign students returned back to their home coun tries

Fig. 1 Graphical representation of participants demography 
Participants' general knowledge about coronavirus was also assessed during the survey. Vast majority of the participants $(90 \%, 534)$ were aware of the incubation period of the coronavirus (1-14 days) and knew that 40+ individuals were under high risk when compared to younger individuals $(82 \%, 487)$. When asked about the symptoms of COVID-19, majority of participants reported fever $(97 \%, 576)$, dry-cough $(94 \%, 558)$, and shortness of breath $(97 \%, 576)$ as symptoms. One-third of the participants reported headache $(34 \%, 202)$ and diarrhea $(35 \%$, $208)$, two-third of the participants reported sore throat (64\%, 380 ) as symptom of the disease. Less than $20 \%$ of the participants selected runny nose and dizziness as symptoms of COVID-19. When asked about the measures for preventing COVID-19, majority of the participants reported that cleaning hands (94\%, $558)$, use of gloves $(82 \%, 487)$, use of face masks $(89 \%, 529)$, not going out unless needed $(98 \%, 582)$, avoiding family and neighbor visits $(94 \%, 558)$, maintaining social distance $(98 \%, 582)$, disinfecting clothes worn outside $(85 \%, 505)$ can help to prevent transmission of COVID-19. When asked about the transmission of COVID-19 via hand contact, $73 \%$ (434) of the participant confirmed that. COVID-19 transmission type was also asked to the individuals and 75\% (446) of the participants knew that the disease is zoonotic. The present study reported no significant relationship between participants' responses with gender, age, or home country. The study also indicated a significant relationship between participants' education level (whether they have taken microbiology courses) and responses to questions like transmission routes, whether the disease is zoonotic or not.

\section{Measurement of COVID-19 Precautions in the Eye of Dentists}

Almost all of the participants $98.5 \%$ (585) reported that, dentists are at high-risk group in terms of COVID-19 and measurement of patients' temperatures should be mandatory $(93 \%, 551)$. A total of $89 \%$ (527) students indicated that exceeding social distance limit with patient, exposure to body fluids such as blood or saliva $(94 \%, 562)$ and use of high speed rotary instruments $(54 \%, 325)$ are the causes that increases dentists tendency to the disease. Participants reported that it is important to change gloves $(97.5 \%, 580)$, face masks $(96 \%$, $570)$, wearing face shields $(93 \%, 553)$. They were aware of importance of disinfecting units after treatment of each patient $(90 \%, 538)$ and sterilization of instruments used $(91 \%, 546)$. Majority of participants mentioned that during theCOVID-19 outbreak, painful tooth requiring root canal treatment $(90 \%$,
$535)$, swelling of face $(82 \%, 492)$, and trauma $(87 \%, 518)$ were accepted reasons for patient treatments, whereas, only small percentage of participant population which significantly comprised of pre-clinical students selected dental caries $(14 \%, 88)$, routine control $(2.5 \%, 15)$, and completion treatment started before outbreak $(36 \%, 207)$ as acceptable reasons in treatment. The clinical questions reported statistically significant relationship between participants' responses with their level of study (pre-clinical and clinical students).

\section{Measurement of Perception Levels of Participants on COVID-19}

Participants were asked about the mortality rates of SARSCoV-2 in comparison to some other viral diseases (SARS, MERS, and Seasonal Influenza). One-fourth $(25.9 \%, 202)$ of the individuals reported that mortality related to seasonal influenza is lower than SARS-CoV-2, whereas, 22\% (175) and $16.9 \%$ (132) of the respondents respectively indicated SARS and MERS have lower mortality rates than SARS-CoV-2. Onethird $(271,34.7 \%)$ of the participants indicated that they have no knowledge about the mortality rates. When information about current COVID-19 diagnostic tests such as serological tests and RT-PCR were asked, minor level of participants were aware of the importance of $\operatorname{IgG}(16 \%, 99)$ and $\operatorname{IgM}(20 \%, 122)$ antibody measurements, however, nearly half of them were aware of the importance of RT-PCR analysis $(45 \%, 286)$ in terms of COVID-19 diagnosis. Participants who had selected $\operatorname{IgE}(8.5 \%, 51)$ and $\operatorname{IgA}(12 \%, 76)$ measurements were reported as minorities (Fig 2). However, 49\% (295) of the participants indicated that they had no opinion regarding the question, which were significantly belonging to dental students did not have microbiology course yet (1st year students). Moreover, the participants' knowledge about the speed of the diagnostic tests was also asked. Only 27\% (162) of the participants were aware of the fact that serological tests (measuring antibody levels) were responsible for quicker diagnosis of the disease, but 30\% (182) of the individuals reported RT-PCR analysis is quicker and $43 \%$ (250) of them reported that they had no opinion regarding question. General questions regarding the diagnostic tools were also included in the survey. Nearly, onethird of the participants $(30 \%, 174)$ reported that, RT-PCR analysis could possibly give false-negative result when sample is not taken properly and $10 \%$ (56) of them were aware of that RT-PCR has relatively more accuracy when compared to the serological tests (Fig 2). Some other chosen answers were IgM

Knowledge of participants about COVID-19 diagnostic Tests
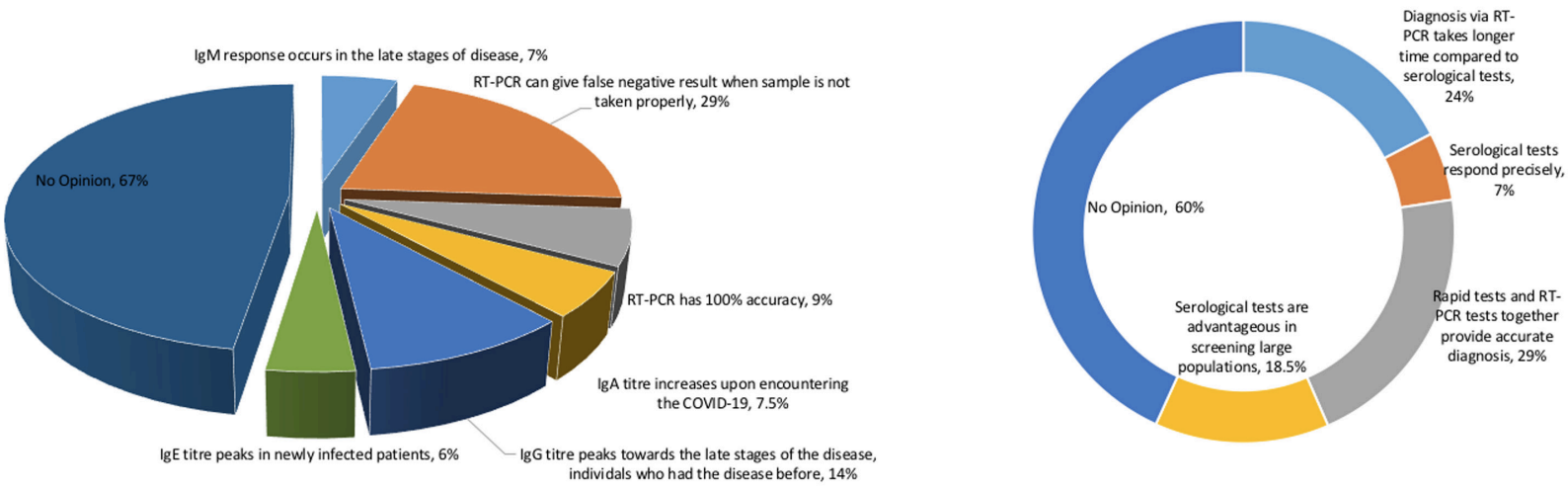

Fig. 1 Graphical representation of participants answers on COVID-19 diagnostic methods. 
response develops in the late stages of disease $(7.2 \%, 43), \operatorname{IgA}$ titer increases upon encountering COVID-19 (7.6\%,45), IgG titer peaks towards late stages of the disease, and individuals who had recovered disease $(13.9 \%, 35)$, IgE response peaks in newly infected individuals $(5.89 \%, 35)$. Two-third $(66.6 \%)$ of the participants had indicated that they had no opinion about the test principles. The present study reported significant relationship between participants' responses on COVID-19specific questions, serological tests etc. and whether participants received microbiology course or not.

\section{Discussion:}

This survey aims to give an insight on the level of knowledge and awareness of dentistry students on COVID-19 who are studying in Cyprus. The data obtained provide important clues when it is assumed that dentists are at the top of the professions with the highest risk group ${ }^{5}$ and $37 \%$ of the participants who responded to the survey were actively working in the clinic. In addition to these, the importance of microbiology classes, the depth of the curriculum and the level of awareness about COVID-19 were determined in line with the responses given by the students of pre-clinical and clinical classes.

Present study covered a total of 594 pre-clinical and clinical dental students with equally distributed male and female populations. International students were asked whether they returned to their home countries and the reason of it. As Cyprus is an island, there are only two ways of transportation, air and sea. Upon confirmation of the first case reported in Cyprus on 10 March, airports and borders were closed on the 16 March. ${ }^{20}$ While leaving of the country were maintained with a controlled manner and foreign citizens living/studying in the country were allowed to return to their homes, only $47 \%$ of the participants returned to their countries, taking into account the difficulties that might be experienced during returns. This might be the main reason why most of the participants stayed in Cyprus. When asked about the reason for leaving Cyprus, the majority of the participants mentioned that they preferred to be with their families during the pandemic and some of them specified the economical issues.

The vast majority of the participants had known the origin of the pandemic was China, the country with highest mortality was USA, and more than half of the participants had known that the highest recovery rate was in China. ${ }^{6}$ This showed, the participants were following the current worldwide status of the COVID-19 and well aware of the situation. The estimated incubation time for COVID-19 was reported to be between 1 and 14 days and older individuals were more susceptible. ${ }^{1,7}$ Study participants knowledge about incubation period of the and susceptibility of the COVID-19 were impressive. This knowledge was very important for participants working in dental clinics in terms of the determination safe period for suspected patients. Knowledge of the participants about common symptoms of the COVID-19 was also investigated in the study. Previous literature indicated that dentists' knowledge on contagiousness of respiratory diseases was limited amongst other health-care professionals..$^{21,22}$ Despite the previous researches, majority of participants were found to be highly informed about the common symptoms of the COVID-19 (dry-cough, high fever, and shortness of breath). ${ }^{9-11}$ The essential actions in disease management were also investigated in the survey. Participants were reported to have a good knowledge in general disease management protocols, for instance; importance of washing hands, use of gloves and face masks, not going out unless necessary, maintaining social distance, avoiding of family and neighbor visits, and disinfecting clothes worn outside.

Some more questions were also added in the survey to measure the knowledge about precautions needed to be taken in the field of dentistry. Nearly all of the participants (99\%) were aware of the fact that dentists are at the top of the professions with highest risk group due to the normal exceeding social distance with patient and exposure to body fluids of patients, with more than half of the individuals were aware of risk created due to the usage of rotary instruments during treatment. ${ }^{5}$. ${ }^{23}$ The individuals who are aware of the risk of using rotary instruments were significantly found to be the ones with clinical experience. These have a good knowledge in rotary instruments as they are actively using them during clinical practices. After asking about the risks that may cause danger in clinic, participants were asked about necessary measures to minimize these risks. The answers received were quite satisfactory. Nearly all of the participants were aware of the importance of using face shields, face masks, gloves, disinfecting units after each treatment, proper sterilization of instruments, measuring patients' temperatures prior to entry to hospital in terms of protecting both patients and dentists. When asked about which condition should be a reason to apply to dentists during pandemic period, vast majority of them have mentioned that patients with painful tooth requiring root canal treatment, patients with swollen face and trauma patients should only apply to dentists. Dental caries and completion of treatment started before pandemic (15\% and 35\%, respectively) were also selected as conditions should be cared during COVID-19 pandemic which can be attributed to their insufficient clinical experiences. Studying at clinical classes significantly affected the answer of the students and only students at pre-clinical classes selected these two choices.

It was aimed to measure the actual knowledge of the students and evaluate its relationship with their education level. Therefore, 'no opinion' option was added to the appropriate questions in the survey for the prevention of random selection among choices. Participants' detailed knowledge about the rate of COVID-19 infection in comparison to other viral diseases, diagnosis methods and immune response formed during and after infection was also investigated during the survey. The mortality rates of SARS-CoV-2, seasonal influenza, SARS$\mathrm{CoV}$, and MERS-CoV were also asked to the participants. More than on-third of the participants knew that, seasonal influenza has lower mortality rate than SARS-CoV-2 whereas SARS and MERS had higher mortality rates. ${ }^{5}$ However, $45 \%$ of the participants, especially those who are first-year students, did not answer this question correctly. The main reason for this may be due to the lack of microbiology classes in the first years of dentistry curriculum. Test methods for COVID-19 diagnosis were also asked during the survey. Nearly half of the participants had knowledge about the importance of RT-PCR tests during disease diagnosis, however only one-fifth of the participants were aware of the importance of antibody titer (IgG and IgM) measurements by serological tests. Nearly half of the participants had no knowledge about the diagnostic tests according to the survey. Further detailed questions were also asked about the diagnostic tests. Only one-fourth of the participants stated that the serological tests respond faster 
than the RT-PCR tests, while the remaining three-fourth either gave wrong answer or had no opinion. The detailed questions were asked against COVID-19 diagnostic tools and about one-third of the participants showed that they knew the importance of sampling while performing RT-PCR tests, and that if the sample was not taken properly, false-negative data might be obtained. One in ten participants stated that RT-PCR had high accuracy as well. In addition to these correct answers, ${ }^{24,25}$ approximately two-third of the participants stated that they did not know about this question. Nearly one-third of the participants are aware that serological tests and RT-PCR tests together provide more accurate results and one-fifth of them indicated that quick-serological tests are advantageous in screening large populations. However, more than half of the students, especially the ones in early years of education had no opinion about these aspects of the COVID19 diagnostic tests. These might be attributed to condense immunological and pharmacology classes are held during the second and third years of the curriculum.

Dental students studying in Cyprus showed high awareness of COVID-19 and the higher risks of COVID-19 exposure that dental professionals face. Preventing the spread of corona virus during dental applications and the conditions that require treatment during pandemic was dependent on the clinical experience of participants. Having microbiology courses significantly affected the answers to the questions on diagnosis methods and immune response to COVID-19.

\section{Conflicts of Interest Disclosure:}

\section{All authors declare no conflicts of interest.}

\section{References:}

1. Li Q, Guan X, Wu P, Wang X, Zhou L, Tong Y, et al. Early transmission dynamics in Wuhan, China, of novelcoronavirus-infected pneumonia. N Engl J Med 2020 Mar 26;382(13):1199-1207. Doi: 10.1056/NEJMoa2001316

2. World HealthOrganization. WHO Timeline - COVID-19 [accessed 15 June 2020]. https://www.who.int/news-room/detail/27-04-2020-who-timeline--covid-19

3. Chan JF, Yuan S, Kok KH, To KK, Chu H, Yang J, Xing F, Liu J, Yip CC, Poon RW, et al. A familial cluster of pneumonia associated with the 2019 novel coronavirus indicating person-to-person transmission: a study of a family cluster. Lancet. 2020;395(10223):514-523.

4. Lu R, Zhao X, Li J, Niu P, Yang B, Wu H, Wang W, Song H, Huang B, Zhu $\mathrm{N}$, et al. Genomic characterisation and epidemiology of 2019 nove coronavirus: Implications for virüs origins and receptor binding. Lancet 2020;395(10224):565-574.

5. Meng L, Hua F, Bian Z. Coronavirus Disease 2019 (COVID-19): Emerging and future challenges for dental and oral medicine. J Dental Res. 2020;99(5):481487. Doi:10.1177/0022034520914246

6. Worldometer. 2020. COVID-19 CoronavirusPandemic. [Accessed 15 June 2020]. https://www.worldometers.info/coronavirus/

7. Backer JA, Klinkenberg D, Wallinga J. Incubationperiod of 2019 novelcoronavirus (2019-nCoV) infections among travellers from Wuhan, China, 20-28 January 2020. Euro Surveill. 2020;25(5). Doi:10.2807/15607917. ES.2020.2825.2805.2000062.
8. Holshue ML, DeBolt C, Lindquist S, Lofy KH, Wiesman J, Bruce H, Spitters C, Ericson K, Wilkerson S, Tural A, et al. First case of 2019 novelcoronavirus in the United States. N Engl J Med 2020. Doi:10.1056/NEJMoa2001191.

9. Hejaz HA, Zalloum A, Attili R. Novel Coronavirus Disease-2019: Epidemiology, diagnosis, therapeutics and guideline protocol for disease management in different countries. Iraq Med J. 2020 Jun 26;4(2).

10. Guan W-J, Ni Z-Y, Hu Y, Liang W-H, Ou C-Q, He J-X, Liu L, Shan H, Lei C-L, Hui DS, et al. Clinical characteristics of 2019 novel coronavirus infection in China. medRxiv2020. Doi:10.1101/2020.1102.1106.20020974

11. Zhou F, Yu T, Du R, Fan G, Liu Y, Liu Z, et al. Clinical course and risk factors for mortality of adult in patients with COVID-19 in Wuhan, China: A retrospective cohort study. The Lancet 2020;395(10229):1054-1062. Doi: https://doi.org/10.1016/ S0140-6736(20)30566-3

12. Saleh M, Saleh M, Zahid MN. Awareness and response to Coronavirus Disease (Covid-19) epidemic among the Arab population in the Eastern Mediterranean Region and Arab Peninsula. J Contemp Med Sci. 2020 Aug 26;6(4).

13. Wang D, Hu B, Hu C, Zhu F, Liu X, Zhang J, Wang B, Xiang H, Cheng Z, Xiong Y, et al. Clinical characteristics of 138 hospitalized patients with 2019 novel coronavirus-infected pneumonia in Wuhan, China. JAMA 2020. Doi:10.1001/ jama.2020.1585.

14. Kui L, Fang YY, Deng Y, Liu W, Wang MF, Ma JP, Xiao W, Wang YN, Zhong $\mathrm{MH}, \mathrm{Li} \mathrm{CH}$, et al. Clinical characteristics of novel coronavirus cases in tertiary hospitals in Hubei province. Chin Med J (Engl) 2020. Doi:10.1097/ CM1099.0000000000000744

15. Yang Y, Lu Q, Liu M, Wang Y, Zhang A, Jalali N, Dean N, Longini I, Halloran $M E, X u B$, et al. Epidemiological and clinical features of the 2019 novel coronavirus outbreak in China. J Dental Res. 2020. Doi:10.1101/2020.1102 .1110 .20021675 .

16. World Health Organization. Clinical management of severe acutere spiratory infection when novel coronavirus (2019-nCoV) infection is suspected: Interim guidance [accessed 15 June 2020]. 2020. https://www.who.int/ publications-detail/clinical-management-of-severe-acute-respiratoryinfection-when-novel-coronavirus-(ncov)-infection-is-suspected.

17. Secon H. Nearly 3,400 Chinese healthcare workers have gotten the coronavirus, and 13 have died. Business Insider 2020 Mar 04.

18. Rothe C, Schunk M, Sothmann P, Bretzel G, Froeschl G, Wallrauch C, Zimmer T, Thiel V, Janke C, Guggemos W, et al. Transmission of 2019-nCoV infection from an asymptomatic contact in Germany. N Engl J Med. 2020. Doi:10.1056/NEJMc2001468

19. Spagnuolo G, De Vito D, Rengo S, Tatullo M. COVID-19 outbreak: An overview on dentistry. Int J Environ Res Public Health 2020;17(6):2094. Doi: https://doi.org/10.3390/ijerph17062094

20. TRNC Public Information Office. Is North Cyprus set to become first country to eradicate COVID-19? [Accessed 15 June 2020]. https://pio.mfa.gov.ct.tr/ en/is-north-cyprus-set-to-become-first-country-to-eradicate-covid-19/

21. Baseer M, Ansari S, AlShamrani S, Alakras A, Mahrous R, Alenazi A. Awareness of droplet and airborne isolation precautions among dental health professionals during the outbreak of coronavirus infection in Riyadh city, Saudi Arabia. J ClinExpDent 2016 Oct;8(4):e379-e387. Doi: 10.4317/ jced.52811

22. Abolfotouh MA, AlQarni AA, Al-Ghamdi SM, Salam M, Al-Assiri MH, Balkhy $\mathrm{HH}$. An assessment of thelevel of concern among hospital-based healthcare workers regarding MERS outbreaks in Saudi Arabia. BMC InfectDis 2017 Jan 03;17(1):4. Doi: 10.1186/s12879-016-2096-8

23. Centers for Disease Control and Prevention 2020. CDC recommendation: postponenon-urgent dental procedures, surgeries, andvisits. [Accessed 15 June 2020]. https://www.cdc.gov/oralhealth/infectioncontrol/statementCOVID.html

24. Winter AK, Hegde ST. The important role of serologyfor COVID-19 control. The Lancet Infectious Diseases 2020. Doi: https://doi.org/10.1016/S14733099(20)30322-4

25. Long Q, Liu B, Deng H, Wu G-C, Deng K, Chen Y-K. et al. Antibody responses to SARS-CoV-2 in patients with COVID-19. Nat Med 2020. https://doi. org/10.1038/s41591-020-0897-1

This work is licensed under a Creative Commons Attribution-NonCommercial 3.0 Unported License which allows users to read, copy, distribute and make derivative works for non-commercial purposes from the material, as long as the author of the original work is cited properly. 